Y.-H. Chu, N.B. Suntzeff, J.E. Hesser, and D.A. Bohlender, eds.

\title{
Relaxation of Star Clusters and Color Gradients
}

\author{
Christian Boily ${ }^{1}$ \\ Queen Mary $\mathcal{B}$ Westfield College, University of London \\ Mile End Road E1 4 NS, England.
}

\begin{abstract}
Large interstellar distances imply that protostars are dynamically decoupled from gas ram pressure (see, e.g., Aarseth, Lin \& Papaloizou 1988) and are effectively isolated self-gravitating objects. With this in mind we apply Newtonian gravity to collapsing cold spheroids of point masses to model the Lin-Mestel-Shu (1965) solution for dust. Incomplete violent relaxation (e.g., van Albada 1982) means that traces of the initial orbit distribution in energy remain. We show here that furthermore the degree of orbit mixing achieved correlates well with initial morphology, by collapsing two-mass component uniform spheroids of different aspect ratio. We setup a test problem to seek out a relation between orbit survival and initial morphology which we then solve numerically.
\end{abstract}

\section{N-Body Integration.}

Our toy model consists of a uniform sphere containing two mass components, one inside, the other outside, radius $r=\pi$; the bounding radius $=5$. Each mass bin is sampled with 5,000 bodies. With $m_{1} / m_{2}=1 / 3$ the mean density is everywhere the same, hence the free-fall time is unique. With the more massive stars confined to $\pi<r<5$, the intention is to favour mass ejection from, or otherwise disturb, the much lighter central distribution. This is a robust test of orbit survival in energy space under violent relaxation.

The 10,000-particle simulation was performed with the code NBODY1 on the HARP computer. The length $l$ of the smoothed potential $=3 \times 10^{-4}$ (in system units), well below close-encounter deflection impact-parameter values (see Boily et al. 1999 [BCM]; Theis \& Spurzem 1998). The simulation ran for a total of 6 initial crossing times. A small velocity was given to each star such that the virial ratio $Q \equiv-T / W=1 / 200$ initially. Two more simulations were performed with the same random seed distributions, but now flattening the zaxis by factors of 2 (hamburger) and 10 (pancake): this covers an appropriate range of spheroid aspect ratios.

The short timescale of the simulations matches age estimates of the youngest LMC star clusters (Elson et al. 1987).

\footnotetext{
${ }^{1}$ Now at: Astronomisches Rechen-Institut, Mönchhofstrasse 12-14, Heidelberg D-69120, Germany.
} 
We measured orbit-mixing by monitoring in time the ranking $\mathcal{R}_{t, i}$ of each $\left(i^{\text {th }}\right)$ star relative to one another, introducing

$$
\Delta \equiv\left(\frac{1}{2 N} \sum_{S} \sum_{i_{\mathrm{bin}}}\left[\mathcal{R}_{t, i}-\mathcal{R}_{o, i}\right]^{2}\right)^{1 / 2}
$$

Completely mixed orbits give $\Delta \simeq 57.7$ when the number of rank bins $S=200$.

\section{Discussion.}

We found that while both spherical and 'pancake' collapses erase memory of the initial vertical $(z)$ structure rather well, this is not so for the 'hamburger' simulation (aspect ratio $=1 / 2$ ): we find post-collapse $\Delta_{z} \approx 42$, well below the expected value for fully-mixed systems.

The discovery of young star clusters with color gradients (see Fischer et al. 1998; Hunter et al. 1998; Kontizas et al. 1998) suggests stellar formation processes may have been at work throughout their formation history. When star formation proceeds on a timescale short compared with a cluster free-fall time, color (mass) gradients may develop early on and not be deleted by violent relaxation. Memory of the initial particle distribution is not completely obliterated by cold collapse, particularly when collapse proceeds from a distribution of initial aspect ratio $\sim 1 / 2$.

Combining this result with the findings of BCM, we would anticipate color gradients be present in roundish clusters, and absent for those with large (on the mean) ellipticities. These results obtained for axisymmetric distributions are to be applied cautiously, given that (young) LMC star clusters are likely triaxial (e.g., Han \& Ryden 1994).

Acknowledgments. Warm thanks to C.J. Clarke and S.D. Murray for discussions, and the organisers of the conference for agreeing to this (last minute) contribution.

\section{References}

Aarseth, S.J., Lin, D.N.C., \& Papaloizou, J.C.B. 1988, ApJ, 324, 288

Boily, C.M., Clarke, C.J., \& Murray, S.D. 1999, MNRAS, 302, 339

Elson, R.A.W., et al. 1987, ApJ, 323, 54

Fischer, P., et al. 1998, AJ, 115, 92

Han, C.H., \& Ryden, B.S. 1994, ApJ, 433, 80

Hunter, D.A., et al. 1998, ApJ, 478, 124

Kontizas., M., et al. 1998, A\&A, 336, 503

Lin, C.C., Mestel, L., \& Shu, F.H. 1965, ApJ, 142, 1431

Theis, C., \& Spurzem, R. 1998, A\&A, 341, 361

van Albada, T.S. 1982, MNRAS, 201, 939 